\title{
POLITICAS EDUCATIVAS DE LOS GOBIERNOS LIBERALES EN LA CREACIÓN Y ORGANIZACIÓN DE LAS ESCUELAS NORMALES DEL DEPARTAMENTO DE BOYACÁ (1930-1938) ${ }^{1}$
}

\author{
DOI: http://dx.doi.org/10.1590/2236-3459/57787
}

\author{
Mónica Liset Valbuena Porras \\ Universidad Pedagógica y Tecnológica de Colombia, Colombia.
}

\section{$\cos 80$}

\begin{abstract}
Resumen
El departamento de Boyacá quien, a comienzos del siglo 20, concentro más de la mitad de la población en zonas rurales, se convirtió en pionero de la construcción de escuelas y mejoras en las condiciones de los docentes, entre las que estuvo la apertura de escuelas Normales que permitió formar adecuadamente a los maestros y maestras del departamento entre 1930-1938. Teniendo en cuenta lo anterior, se formularon 4 ejes fundamentales para el desarrollo de la investigación: Las políticas educativas nacionales implementadas en las Escuelas Normales de Colombia, la puesta en práctica de estas reformas educativas en el departamento Boyacá, la capacitación de maestros, los pensum implementados en estas instituciones y los procesos de escolarización desarrollados durante el periodo de estudio. Se plantea como pregunta: ¿Cómo incidieron las políticas educativas de los gobiernos liberales en la creación y organización de las escuelas normales en Boyacá? Se sustenta desde el método de historia social de la educación y la metodología es de tipo hermenéutico para la recolección y análisis de las fuentes consultadas como memorias de ministros de educación de la época, secretarios de educación, periódicos.
\end{abstract}

Palabras clave: políticas educativas, gobiernos liberales, escuelas normales, formación.

\section{AS POLÍTICAS EDUCACIONAIS DOS GOVERNOS LIBERAIS NA CRIAÇÃO E ORGANIZAÇÃO DAS ESCOLAS NORMAIS DE BOYACA (1930-1938)}

\begin{abstract}
Resumo
O departamento de Boyacá que, no início do século 20, concentrou mais da metade da população em áreas rurais, tornou-se um pioneiro na construção de escolas e na melhoria das condições dos professores, entre as quais tivemos abertura de escolas normais, que permitiram a formação adequada dos docentes do departamento, entre 1930-1938. Tendo em conta o acima exposto, foram formulados 4 eixos fundamentais para o desenvolvimento da pesquisa: As Políticas nacionais de educação implementadas nas escolas normais da Colômbia, a prática destas reformas educacionais no departamento de Boyacá, a formação de professores, os currículos desenvolvidos nessas instituições e os processos de escolarização desenvolvidos durante o período do estudo. Apresenta-se como questionamento: Como as políticas educacionais dos governos
\end{abstract}

\footnotetext{
${ }^{1}$ Este artículo es resultado del proyecto de investigación: Maestras y escuelas normales en Colombia durante los siglos 19 y 20, desarrollado por el grupo de investigación Historia y Prospectiva de la Universidad Latinoamericana - Hisula. Financiado por la Dirección de Investigaciones de la Universidad Pedagógica y Tecnológica de Colombia a través de estímulos jóvenes investigadores Colciencias 2014. Actualmente docente de la Escuela de Ciencias Sociales, artes y humanidades de la universidad Nacional Abierta y a Distancia-UNAD.
} 
liberais influenciaram na criação e organização das escolas normais em Boyacá? A pesquisa apoia-se no método da história social da educação e na metodologia de tipo hermenêutica para selecionar e analisar as fontes consultadas, como memórias de ministros de educação da época, secretários de educação.

Palavras-chave: políticas educacionais, governo liberal, escolas normais, formação.

\title{
EDUCATIONAL POLICIES OF LIBERAL GOVERNMENTS IN THE CREATION AND ORGANIZATION OF THE NORMAL SCHOOLS OF BOYACA (1930-1938)
}

\begin{abstract}
At the beginning of the 20th century, most of Boyacá's population was in the rural areas becoming this department in a pioneer for building schools and improving the teacher's work conditions; as part of this, new Normal schools were opened, which allowed to train new teachers between 1930 and 1938. Taking it into account, 4 key central concepts were formulated to develop this research project: National educational policies implemented in the Colombian Normal Schools, the implementation of these educational reforms in the department of Boyacá, the teacher's training, curriculum implemented in these institutions and the schooling processes developed during the study period. The following question was considered: How did the educational policies of liberalism influence on the creation and organization of Normal schools in Boyacá? It is supported by the social history method of education; for the data collection and analysis of sources such as: memoirs of education ministers of that time, secretaries of education and newspapers, the hermeneutical approach methodology was used.

Keywords: educational policies, liberal governments, normal schools, formation.
\end{abstract}

\section{LES POLITIQUES ÉDUCATIVES DES GOUVERNEMENTS LIBÉRAUX DANS LA CRÉATION ET L'ORGANISATION DES ÉCOLES NORMALES DE BOYACA (1930-1938)}

\section{Résumé}

Le département Boyaca qui, au début du 20 e siècle, concentre plus de la moitié de la population dans les zones rurales, est devenu un pionnier de la construction d'écoles et d'améliorer les conditions des enseignants, parmi lesquels se trouvait l'ouverture d'écoles régulières qui enabled bien former les enseignants du département entre 1930-1938. les politiques éducatives nationales mises en œuvre dans les écoles normales de la Colombie, la mise en œuvre de ces réformes éducatives dans le département de Boyacá, la formation des enseignants, programmes: Étant donné les, 4 axes fondamentaux ci-dessus pour le développement de la recherche ont été formulées mis en œuvre dans ces institutions et les processus développés scolarité pendant la période d'étude. Elle pose comme question: Comment influencé les politiques éducatives des gouvernements libéraux dans la création et l'organisation des écoles normales à Boyaca? II est soutenu par la méthode de l'histoire sociale de l'éducation et de la méthodologie herméneutique pour recueillir et analyser les sources consultées comme souvenirs des ministres de l'éducation de l'époque, les secrétaires de l'éducation, de type régulier.

Mots-clés: les politiques éducatives, les gouvernements libéraux, écoles normales, la formation. 


\section{Introducción}

nalizar la educación en Colombia, nos lleva abordar el surgimiento de las
escuelas normales, en la segunda mitad del siglo XIX, cuando las olas a
favor de la formación de mujeres iniciado en Europa y los Estados Unidos, favoreció el surgimiento de estas, y su consolidación hacia 1870. Estas instituciones funcionaron bajo el rigor de la disciplina, pero su impacto, se logró en 1877 al crearse instituciones anexas: una elemental y otra superior. Este buen funcionamiento y cubrimiento de las demandas, logró su consolidación a través de los informes anuales, que hicieron notar su importante tarea en la formación de mujeres.

Con la entrada del siglo XX, se dieron los primeros procesos de Modernización a través de las reformas llevadas a cabo con ley 39 de 1903 y el decreto reglamentario 491 de 1904, los cuales se retomaron a partir de 1918, cuando se planteó la necesidad de una política educativa acorde a los retos del momento.

Por ello, las misiones alemanas convocadas desde 1924 generaron los primeros cambios educativos, los cuales fueron implementados por Rafael Bernal Jiménez en el Departamento de Boyacá, y Agustín Nieto Caballero², quien un año después trajo de visita al educador Decroly al Gimnasio Moderno ${ }^{3}$, el cual ayudó al desarrollo de la Escuela Nueva ${ }^{4}$

2 Nieto Caballero además de fundar una institución privada para experimentar las tendencias modernas en pedagogía, incidió también en el proceso de reforma educativa que se inicia en la década del veinte; así, fue uno de los ideólogos de la decisión tomada en el año de 1924, durante el gobierno del conservador Pedro Nel Ospina, de contratar la misión pedagógica alemana que se encargaría de elaborar un proyecto de reforma educativa nacional. Este proyecto, aunque no haya tenido aplicaciones inmediatas debido a las presiones políticas y religiosas, trazó los ejes generales sobre la reforma educativa Nacional que serán retomados por gobiernos posteriores. Ver Herrera (1999, p. 74). En 1925 Agustín Nieto Caballero respondió a esos deseos al invitar a Ovide Decroly a una visita al Gimnasio Moderno, para exponer sus concepciones sobre la Escuela Nueva. Anticlerical notorio el médico Belga había trabajado primero en neurología, después se especializó en la educación de niños anormales para los cuales fundó un instituto, y más tarde se orientó a la educación general. Según Decroly, el mayor error de los métodos tradicionales consistía en considerar a los niños como adultos en miniatura y no como niños integrales; por ello recurrían a la memorización y a una disciplina excesiva que volvía pasivo a los alumnos. Planteó una pedagogía en tres etapas: la Observación por el niño de la realidad y su contorno; la asociación de estas asociaciones y la teoría y la expresión tanto oral como escrita o manual de los conocimientos adquiridos a partir de las dos primeras fases. Centros de interés orientados hacia las actividades esenciales del hombre - la alimentación, trabajo, vestido, por ejemplo - permitían concretar esta pedagogía. Finalmente, mediante el método de la globalización, la enseñanza estimulaba el interés de los alumnos hacia materias más escolares. Las conferencias de Decroly en el Gimnasio Moderno atrajeron a los pedagogos de todo el país. Originaron los primeros centros de interés en algunas escuelas privadas, antes de la adopción oficial del método en 1936. Ver MEN. El doctor Decroly en Colombia. HELG, Aline. La educación en Colombia 1918-1957. Bogotá: Universidad Pedagógica Nacional, 2001.

${ }^{3}$ El Gimnasio Moderno fue una iniciativa pedagógica del Señor Agustín Caballero para el año de 1914 convirtiéndose luego en el escenario de conferencias dictadas por el profesor Decroly. El Gimnasio Moderno, establecido cerca de Bogotá, distante de toda vía de comunicación internacional, solo podría contar en sus comienzos con alumnos de una población relativamente pequeña, 200.000 habitantes, y en la que solo se encuentra un reducido número de personas capaces de comprender el sentido de una EN y sobre todo, capaces de asociar a ella de una manera útil y provechosa [...]. El Gimnasio Moderno debe ser clasificado entre las Escuelas Nuevas, pues exhibe los principales caracteres de ella: el estar situado en el campo, los métodos activos de instrucción, la disciplina de confianza, la importancia que dan a la educación física y moral, a los trabajos manuales, a las excursiones, a las obras sociales, etc. Ver Inspección Nacional de Educación Primaria y Normalista. Bogotá: Imprenta Nacional, 1932, p. 9-32.

${ }^{4}$ Tuvo como principio "facilitar la individualización del estudiante dentro de la colectividad en un ambiente sano propicio para el trabajo", el cual contribuye al desarrollo integral del niño y el maestro como hacedor de experiencias para hacer más atractiva la enseñanza debe sacar ampliamente partido de proyecciones, trabajos manuales, colecciones, excursiones, viajes para hallar en ellos elementos que necesite". Ver BÁEZ, Lina Adriana Parra. Decroly y Boom, iniciación general del método Decroly: ensayos de aplicación a la

ก. 52

Maio/ago., 2017

p. 194-213 
en Colombia. El balance hecho por estás misiones, permitió encontrar los elementos que impidieron los procesos de cambio en la educación nacional, entre los que se destacó: el cambio de ministros constantemente, el nombramiento de maestros, muchos de ellos nombrados por relaciones políticas, alza de salarios, falta de capacitaciones a los maestros, que trajo como resultado la ley de 1927 que recogió las recomendaciones de las misiones alemanas.

Con la llegada de los gobiernos liberales, después de 45 años de estar ausentes del poder, tuvieron que enfrentar las convulsiones internacionales, luchas sociales, disputas generadas entre la iglesia y el Partido Conservador, defensores de una educación católica y moralizante; procesos que no obstaculizaron, la puesta en marcha de reformas y programas educativos por unificar la educación urbana y rural, creación de establecimientos de primaria y secundaria, facultades de educación con la aplicación de métodos pedagógicos de la escuela activa europea, campañas de cultura aldeana, capacitación vacacional de maestros y maestras.

\section{Políticas educativas implementadas por los gobiernos liberales en Colombia: una puerta inconclusa a la modernización}

El periodo de la República Liberal como iniciador de la modernización de la educación ${ }^{5}$ en Colombia, se dio en medio de convulsiones internacionales: La primera y segunda guerra mundial, el auge del autoritarismo en diversos países europeos y la intervención de Estados Unidos en territorio Latinoamericano.

Igualmente, el país atravesó por una serie de luchas sociales: la organización del magisterio, lucha de campesinos e indígenas por reformas agrarias, exigencias que llevaron a cabo las mujeres en todo el país, para el reconocimiento de sus derechos civiles, culturales y políticos, que permitiera su desempeño en otros campos de la sociedad, que no estuvieran ligados a su condición materna, que muchas veces la iglesia y el partido conservador buscaron defender y mantener.

Los gobiernos liberales enfrentaron a la iglesia y el Partido Conservador, el cual por mucho tiempo había sido el defensor de una educación Católica, promovida y supervisada por los mismos religiosos:

\footnotetext{
El ministerio de Educación Nacional tuvo que enfrentarse con las resistencias de una sociedad d que aún se movía bajo pautas localistas y partidistas, con un funcionamiento de la educación que llevaba por tradición la ausencia de preocupación estatal, reinando en vez de ésta los intereses privados y el afán mercantilista. A pesar de lo planteamientos que propendían por impartir uniformidad en materia educativa desde este organismo, la mayoría de los ministros tuvieron una gestión administrativa efímera. (HERRERA, 1999, p. 95)
}

Sin embargo, llevaron a cabo campañas culturales para fomentar la creación de bibliotecas, incrementando las adquisiciones y el número de lectores, promovieron la enseñanza técnica, para los sectores populares, las escuelas de artes, oficios, industrias y la educación secundaria. Para la educación superior, busco desarrollar programas que

\footnotetext{
escuela primaria. Revista Historia de la Educación Latinoamericana, n. 6, 2008, p. 168.

${ }^{5}$ Los procesos de modernización presentados en las primeras décadas del siglo 20 fueron acompañados del surgimiento de nuevos sectores sociales y nuevos grupos políticos, así como la redefinición del bloque de poder y su representación en el estado. Ver Herrera (1999, p. 131).
} 
promovió la investigación científica, social y académica, de donde salió los nuevos equipos de gobierno los cuales tuvieron a su cargo la organización de programas acordes a las necesidades e intereses de la población.

Con la llegada al poder de Olaya Herrera como presidente de la República por parte del Partido Liberal, se dio inicio a la Concentración Nacional ${ }^{6}$, los cuales "se dedicaron a conjurar los efectos de la crisis económica y luego a realizar reformas políticas y Sociales, muchas de ellas iniciadas en los gobiernos antecesores, pero siempre aplazadas y frustradas por el predominio de fuerzas tradicionalistas y adversas al cambio" (URIBE, 1989, p. 87). Aprovechando los intentos de transformación del sistema educativo, impulsados por el General Pedro Nel Ospina en 1923, cuando trajo la primera misión belga del educador Octavio Decroly ${ }^{7}$ que fracaso por la oposición de la Iglesia.

Este gobierno tuvo que enfrentar

\begin{abstract}
una tasa de analfabetismo de $63 \%$ de la población en edad escolar, escuelas Normales insuficientes y bajo nivel de preparación del magisterio; planes de estudio y métodos de enseñanza anticuados y rutinarios, tanto en la escuela elemental, como en la enseñanza Secundaria y en la Universidad, la escuela rural cubría sectores mínimos de la población campesina, la inspección escolar apenas si existía y el sistema educativo en general estaba prácticamente a cargo de las instituciones religiosas. (URIBE, 1989, p. 87)
\end{abstract}

Con la crisis financiera del 1929 , muchos de los cambios propuestos no se llevaron a cabo, pero logro establecer un mínimo de reorganización de las escuelas y enseñanza media, creo la Inspección Nacional Educativa para las Escuelas de Primaria, Bachillerato y establecimientos de educación profesional, busco mejorar la capacitación y formación de los maestros con la creación de la Facultad de Ciencias de la Educación ${ }^{8}$ y fomento el desarrollo de la educación Privada9: Instituciones y Universidades dirigidas por comunidades religiosas.

Durante el gobierno de Alfonso López Pumarejo, se realizó profundos cambios en la educación, se crearon programas económicos y sociales, debido a que enfrentaron el inicio del desarrollo industrial, que trajo nuevas exigencias al país, las cuales no cubrieron la educación "por falta de verdaderos maestros en la enseñanza primaria y secundaria. Se propuso a aumentar el presupuesto del estado hacia la educación en un 10\% con la ley 12 de 1934; pasando de 1.920 .000 pesos, es decir $2.6 \%$ del presupuesto nacional para ese año a 7.609 .000 pesos para 1938, cuadruplicando la cuantía a un $8 \%$ del presupuesto nacional" (URIBE, 1989, p. 91).

Sumado a lo anterior,

\footnotetext{
${ }^{6}$ Se llamó Concentración Nacional a la unión de conservadores y liberales que apostaron a un cambio educativo para la nación Colombiana.

7 Desde 1924 el pedagogo Rafael Bernal, impulso los primeros cambios en la educación del departamento de Boyacá: creo Bibliotecas, sistemas médicos escolares y experimentos pedagógicos, para mejorar la escuela rural, teniendo resonancia en otros departamentos como Cundinamarca, Tolima, Antioquia y Nariño. Ver más en Mejía (1989).

${ }^{8}$ Fue transformada en Escuela Normal Superior y trasladada a Bogotá bajo la dirección del Ministerio de Educación Nacional.

${ }^{9}$ Estás instituciones iniciaron su aparición hacia 1932, por los conflictos de concepción y cambios educativos que introdujo el gobierno liberal, los cuales chocaron con la educación tradicional y valores cristianos profesados y defendidos por la iglesia Católica.
} 
introdujo el artículo 14 en la reforma constitucional de 1936, que autorizaba al estado para que, respetando el concepto de libertad de enseñanza, el gobierno pudiera intervenir en la marcha de la educación pública y privada, a fin de garantizar los fines sociales, de la cultura y la mejor preparación intelectual, moral y física de los educandos Igualmente organizó y unificó la escuela rural y urbana, la enseñanza media y universitaria, estableció políticas de formación de maestros /maestras y el acceso de la mujer a todos los niveles educativos que le permitió llegar a todos los ámbitos de una sociedad que por mucho tiempo la invisibilizó como sujeto transformador. (URIBE, 1989, p. 93)

Para mejorar las condiciones de la población en edad escolar, que asistió a las escuelas rurales y campesinas "propuso programas de cobertura en materiales para las escuelas y servicios higiénicos para escolares. Además, considero de urgencia el desarrollo de las escuelas rurales y campesinas frente a las urbanas" (URIBE, 1989, p. 96), a través de la implementación de cartillas y métodos provenientes de la Escuela Nueva, que originó problemas con las autoridades eclesiásticas y locales, quienes interpretaron estás medidas como socialistas.

Durante el periodo de los gobiernos liberales 1930-1938 se llevaron a cabo varias reformas educativas ${ }^{10}$ a nivel nacional que no solo modificaron los planes de estudios de las Escuelas Normales sino las políticas implementadas a nivel departamental - municipal, favoreciendo la apertura y restructuración física de los establecimientos, capacitación profesional y pedagógica de los maestros que enseñaron en las escuelas normales femeninas y masculinas, así como en las Facultades de educación como se señala a continuación:

- Decreto 865 de $1930^{11}$, estableció las juntas pedagógicas para la aprobación de los textos utilizados por los maestros y las maestras a la hora de impartir clases;

- Decreto número 301 de 1933, planteó las disposiciones orgánicas relativas a la Escuela Normal de Institutores de Tunja;

- Decreto número 725 de 1933, dirigido a las Escuelas Normales Departamentales que establezcan el curso de información, en el sentido que los maestros y maestras que estudien en el citado curso se considerarán en servicio en aquel puesto que ocupaban cuando obtuvieron la beca;

- Decreto número 1379 de 1934, propuso la necesidad de la especialización en la Escuela Normal de Tunja hasta que tome el carácter de facultad destinada a la formación del profesorado para la enseñanza secundaria y a la instrucción normalista superior;

- Decreto 1917 de 1935, reunió en una sola las facultades de educación del país;

- Ley 37 de 1935, reguló las normas de ingreso de las maestras y maestros al magisterio;

\footnotetext{
${ }^{10}$ El propósito de la reforma educativa iba más allá de la educación institucionalizada, cubriendo el conjunto de las esferas sociales, y la mayoría de la población, motivo por el cual se requería que los programas educativos permitiesen al individuo, desempeñarse como "educador de sus hijos, de sus subordinados, educador en el medio social" Herrera (1999, p. 146).

11 Decreto por el cual se establece algunas disposiciones sobre Instrucción Pública decretándose quienes debían enseñar historia y geografía y los textos deberán ser aprobados por la junta pedagógica como lo establecen los artículos 1 y 2.
} 
- Ley 32 de $1936^{12}$, reglamentó la igualdad de condiciones para el ingreso a los establecimientos de educación;

- Decreto número 142 de 1936, determino que el título de institutor debía provenir y ser expedido por el Ministerio de Educación Nacional;

- Ley 2 de 1937, señaló el sueldo mínimo de los maestros y maestras, las categorías según las cuales ha de quedar clasificado el personal docente, y, el nombramiento de los mismos.

Las anteriores reformas, reorientaron la función de la Escuela Normal como formadora de docentes y su incidencia en las escuelas normales rurales, agrícolas, anexas y regulares de las zonas urbanas y rurales de los departamentos de Colombia. Contribuyó directamente a la vigilancia de la formación e instrucción escolar por medio del inspector escolar, garantizó la profesionalización de maestros, así como el acceso de niñas y niños a todos los niveles de educación, procurando su bienestar individual y colectivo a través del desarrollo e implementación de Restaurantes escolares, Huertas agrícolas, campañas de higiene, entre otras.

Si bien estas políticas no tuvieron la acogida que se esperaba, por los mismos obstáculos ideológicos y tradicionalistas existentes en los municipios y localidades a nivel nacional, su ejecución en departamentos como Boyacá, Cundinamarca y Tolima generaron grandes alcances, como resultado de los ideales progresistas existentes entre las personas que ocuparon las secretarias de educación departamentales, muchos de ellos provenientes de familias de intelectuales, con formación europea.

\section{Las escuelas normales en Boyacá: un avance hacia la formación de maestros y maestras}

A comienzos del siglo XX, Colombia atravesó por una serie de transformaciones sociales, políticas, económicas y culturales: "La transición de un modelo tradicional de la estructura agraria a un modelo de sociedad que se vendría a denominar moderna con características tales como: Creciente proceso de urbanización, creciente desarrollo industria, ampliación de contacto culturales" (BAUTISTA, 2009, p. 38). Con este panorama Nacional, en el departamento de Boyacá ${ }^{13}$, el clero mantiene su poder político-religioso, legitimado por el apoyo al partido Conservador, las costumbres y creencias patriarcales, así como los residuos de violencia bipartidista.

La población ubicada en un $94 \%$ en la zona rural, dificultó el acceso a las escuelas. ${ }^{14}$ Esto se reflejó en la tasa de analfabetismo (en edad escolar desde los 7 años

\footnotetext{
12 Esta ley en su artículo 1. Establece que ningún establecimiento de educación primaria, secundaria o profesional, podrá negarse a admitir alumnos por motivo de nacimiento ilegitimo, diferencias sociales raciales o religiosas.

${ }^{13}$ Boyacá surge como departamento de la República de Colombia a partir de la Constitución Política de 1886. Luego de 4 años, el gobierno nacional, con la ley n. 68, del 20 de noviembre de 1890, le cedió el territorio de Vásquez, actualmente Puerto Boyacá y, por el decreto ejecutivo n. 306 de 1911, varios municipios se le segregaron para constituir la Comisaria de Arauca. Posteriormente los municipios pertenecientes a la Orinoquía formaron la Intendencia de Casanare, territorio que se le anexó nuevamente en 1950.

${ }^{14}$ Los niños y niñas no son enviados a las escuelas, por las grandes distancias que debían recorrer, por la falta de caminos o porque sus padres no lo consideraban de interés y les imponen tareas agrícolas que no dan espera para la manutención de toda la familia.
} 
en adelante) de $48,2 \%$ para los hombres y $51,8 \%$ para las mujeres ${ }^{15}$. Además, sin contar con la poca participación del sector oficial en la educación primaria, medida en términos del número de escuelas, la cual decrece a finales de la primera mitad del siglo $20^{16}$. Está situación determinó la dinámica de la educación en el Departamento de Boyacá, la preocupación por mejorar la capacitación de maestros, que inició con la implementación de las políticas educativas ${ }^{17}$ de los gobiernos liberales, para el fortalecimiento y creación de las Escuelas Normales, Escuelas Normales Rurales ${ }^{18}$, restaurantes, fomentó a las granjas escolares, expediciones, y publicaciones pedagógicas que contribuyeron al quehacer del maestro y la maestra dentro y fuera del salón de clases.

La necesidad por mejorar la infraestructura y enseñanza en las escuelas normales del todo el país ${ }^{19}$ llevó a la institucionalización y reglamentación de la ley 12 del 17 de diciembre de 1934, con este fin se crear las primeras escuelas normales rurales en el Departamento, las cuales estuvieron destinadas a la formación de mujeres provenientes de estrato medio, a través de becas que ofreció el Estado y el Departamento.

Acorde al informe del Secretario de Educación del departamento de Boyacá, para el año de 1931, se resaltó el compromiso que existió por cumplir con el pagó a docentes e inspectores seccionales de escuelas urbanas y rurales, de médicos, auxilio para las excursiones escolares, propaganda pedagógica, mobiliario, todo tomado del presupuesto del $30 \%$ de la renta de fermentos "en el año existieron 700 escuelas Primarias, así: 680 oficiales y 20 privadas, con un total de 39.704 alumnos" (RAMíREZ, 2008, p. 4), destacándose que en la mayoría de las escuelas del departamento existen maestros graduados y otros que sin serlo se han venido capacitando a través de los cursos vacacionales, que se implementaron en el año 1925 por la existencia de maestras sin preparación, que por vocación ejercían en zonas aisladas y apartadas, sin la menor infraestructura y garantías para el mejoramiento escolar. También señaló que los cursos vacacionales llegaron a más de 200 señoritas que hoy orientan la escuela por senderos de mediano acierto y llevan con su pequeña preparación alguna esperanza de mejoramiento escolar.

${ }^{15}$ Cifras tomadas del Censo General de la República de 1938, p. 156.

${ }^{16} \mathrm{~A}$ su vez en este mismo periodo existe un crecimiento en la participación oficial de los maestros a nivel de Primaria. Las cifras pasan de $75.1 \%$ en 1960 a $77.1 \%$ en 1968 para el País. En Boyacá, este crecimiento es algo mayor: los maestros del sector oficial en 1960 constituían el $85,5 \%$ del total de maestros a nivel primaria y en 1968 era de $91,0 \%$, siendo este periodo de gran interés por el aumento del sector privado sobre la educación rural.

${ }^{17}$ El nuevo tratamiento a lo social y, dentro de él, a lo educativo, se insertó dentro del proyecto político de modernización. Desde allí se diseñó una reforma que buscaba modificar las estructuras educativas, con el propósito de incorporar socialmente la mayoría de la población, dentro de una estrategia de control social.

18 Ver los trabajos de DE ORTEGA, Alarcón. La educación rural y la educación fundamental en Colombia. Centro Regional de Educación Fundamental para la América Latina. En línea: http://www.crefal.edu.mx/biblioteca_digital/coleccion_crefal/tesis/alarcon_ortega.pdf; ÁLVAREZ GALLEGO, Alejandro. Y la escuela se hizo necesaria: en busca del sentido actual de la escuela. Bogotá: MagisterioSociedad Colombiana de Pedagogía, 1995.

${ }^{19}$ La enseñanza normalista, huelga decirlo, tiene por objeto la formación de maestros para las escuelas de la República, y, en tal virtud, es necesario orientar todas las actividades en búsqueda de resultados acordes con esta finalidad específica. De allí que sea menester multiplicar las preocupaciones pedagógicas a fin de dotar a los alumnos de cultura básica que necesitaran para el ejercicio responsables de la profesión, adquisición que debe entenderse desde el punto de vista de los conocimientos y de la capacitación para el estudio y la investigación científica, poniéndolos con ello en situación de elevar su nivel cultural sin quedarse en el modesto mínimum de lo que se exige en un programa de escuela primaria. Ministerio de Educación Nacional. Escuelas normales regulares: plan de estudios, programas, reglamento y disposiciones vigentes. Bogotá: Imprenta Nacional, 1946. 
Entre sus justificaciones estuvo la necesidad de realizar una reforma a favor de la restructuración de la educación rural, para que las maestras y los maestros recibieran una formación integral desde las escuelas normales, partiendo de que la mayoría de docentes en edad entre los 20 y 30 años estaban ubicados en zonas rurales, donde la población presentó el mayor porcentaje de personas analfabetas, tomando de partida el gran papel socio educativo, logrado por la Escuela Normal, reorganizada por Julius Sieber ${ }^{20}$, contribuyendo a la creación y mejoramiento de estos establecimientos.

\section{Políticas educativas implementadas durante 1930 a 1938 en el Departamento de Boyacá}

La crisis económica que vivió el país, por la guerra contra el Perú, y los conflictos bipartidistas, ocasionaron a finales de los años 30 , un recorte presupuestal que afecto directamente a muchos de los departamentos de Colombia, quienes se vieron obligados a cerrar Escuelas Normales para hombres y mujeres. Esta situación se debió a que desde comienzos de los años treinta del siglo 20: "La nación, ni los departamentos, ni los municipios tenían claro hasta qué punto llegaban sus responsabilidades. Se presentaron situaciones en que ni el departamento tenía los recursos para financiar el pago y contratación de nuevos maestros, los municipios no tenían los dineros para construir las escuelas y dotarlas" (RAMÍREZ, 2008, p. 11).

El Departamento de Boyacá enfrentó esta situación a través de la creación y fomentó de fondos provenientes de las rentas de licores, acorde a la ordenanza 2 de 1925, de la cual se destinó el "30 por ciento de la parte que corresponde al departamento en la venta de licores fermentados para atender a los gastos exigidos por los nuevos servicios" (MEMORIAS, 1931, p. 7). Solo para los años 1931-1932 el presupuesto destinado a la educación, proveniente de estos fondos paso del $26 \%$ al $32 \%$ los cuales fueron asignados a los siguientes servicios.

\footnotetext{
${ }^{20}$ El profesor alemán Julius Sieber, quien hacía parte de la segunda misión alemana, traída por el Gobierno de Pedro Nel Ospina, para asesorar a diferentes entidades educativas del país. En 1926, el profesor Sieber regentó la Escuela Normal de Varones de Tunja, allí realizó una ardua labor con el fin de formar maestros de alta calidad y dignos de llevar este título. Su modelo tuvo como propósito fundamental crear en ellos una conciencia investigativa como el único camino para modelar la nacionalidad colombiana auténtica y libre de la dependencia cultural.5 De igual forma, buscó ofrecer una educación en valores para los futuros docentes ya que ellos representaban el modelo a seguir y daban testimonio de grandes virtudes morales, cívicas y culturales, aspectos estos que fueron elementos esenciales de la formación integral de los estudiantes y de esta manera, se obtuvo mejor Calidad Docente y un maestro con Espíritu Renovador y Creativo. A partir de la llegada del profesor Julius Sieber, especialmente en el año 1928, se inicia un estudio profundo sobre la reestructuración de la Escuela Normal, adicionando a los programas regulares de dicho plantel un nivel superior, destinado a la formación de profesores especializados para la enseñanza normalista y la educación secundaria o de bachillerato, el cual comenzó en 1928 gracias a la ordenanza No. 10 de la Asamblea Departamental de Boyacá, como Curso Suplementario de Especialización, con un solo alumno José Estiliano Acosta Gómez. Ver Parra (2008, p. 169).
} 
Tabla 1 - Fomento a la educación por medio de las rentas de licores.

\begin{tabular}{l|l}
\hline Dirección de educación & $13,018 \ldots$ \\
\hline Sección de propaganda pedagógica & $1,944 \ldots$ \\
\hline Inspecciones seccionales de educación & $21,320 \ldots$ \\
\hline Subvención al Colegio de Boyacá & $18,000 \ldots$ \\
\hline Instrucción primaria & 350,58565 \\
\hline Taller de artes y labores manuales & $9,566 \ldots$ \\
\hline Auxilios a los establecimientos docentes & $7,900 \ldots$ \\
\hline Becas & $7,930 \ldots$ \\
\hline Escuelas normales & 13,38840 \\
\hline Asistencia médico escolar & $13,000 \ldots$ \\
\hline Formación del catastro & 19,00118 \\
\hline Imprevistos & 2,71294
\end{tabular}

Fuente: Información tomada de Memorias de los secretarios de Educación en Boyacá. Tunja: 1931.

Los esfuerzos por aumentar el presupuesto fiscal, los llevó a mantenerse en los primeros tres puestos, entre los departamentos que más esfuerzos hicieron por responder a las políticas educativas implementadas por los gobiernos Liberales, pese a las contrariedades y disputas ideológicas. A su vez crearon ordenanzas que favorecieron la construcción de establecimientos escolares, sueldos, pensiones, internados, etc ${ }^{32}$, pero que son la respuesta de los esfuerzos departamentales por garantizar el funcionamiento de instituciones Normales tanto en el sector urbano como rural. La "ordenanza 18 de 1937, estableció para los municipios la obligación de aportar para el fondo de construcciones escolares el 7 por 100 del monto de sus rentas" (RUBIANO, 1938, 41).

Sumado a ello, estaban los esfuerzos nacionales, departamentales y locales, por mantener en funcionamiento las Escuelas Normales para señoritas, garantizar el pago de maestros, excursiones y bibliotecas como se muestra a continuación.

Tabla 2 - Presupuestos para la Educación Pública del Departamento de Boyacá.

\begin{tabular}{|c|c|c|c|c|c|c|c|c|c|c|}
\hline \multirow[b]{2}{*}{$\begin{array}{l}\mathscr{g} \\
\stackrel{0}{0} \\
\stackrel{0}{N}\end{array}$} & \multirow[b]{2}{*}{ 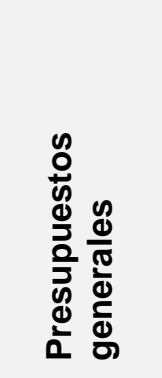 } & \multirow[b]{2}{*}{ 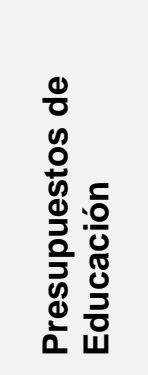 } & \multirow[b]{2}{*}{$\curvearrowright$} & \multicolumn{7}{|c|}{ Distribución de la partida para la educación pública } \\
\hline & & & & 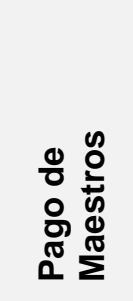 & 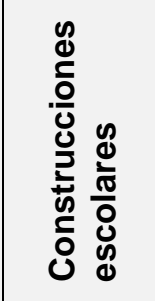 & 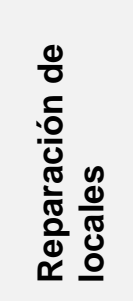 & 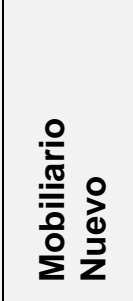 & 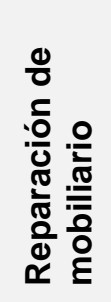 & 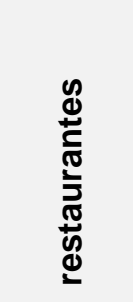 & $\begin{array}{l}\frac{n}{\frac{\pi}{5}} \\
\frac{\pi}{\pi} \\
\text { U⿺ }\end{array}$ \\
\hline $1^{a}$ & 286.29199 & 34.10551 & 11,91 & 5.58300 & 17.42717 & 1.12302 & 1.04500 & 7600 & 4.79064 & 1.04500 \\
\hline $2^{\mathrm{a}}$ & 114.61750 & 20.26884 & 17,64 & 4.38000 & 8.82815 & 1.24943 & 17500 & 37300 & 1.75000 & 35300 \\
\hline $3^{\mathrm{a}}$ & 245.37178 & 20.31299 & 8,2 & 5.54500 & 4.97500 & 1.20288 & 45000 & 1000 & 2.75690 & 9200 \\
\hline
\end{tabular}




\begin{tabular}{|c|c|c|c|c|c|c|c|c|c|c|}
\hline \multirow[b]{2}{*}{$\begin{array}{l}\text { ฮั } \\
\text { c் } \\
\text { N }\end{array}$} & \multirow[b]{2}{*}{ 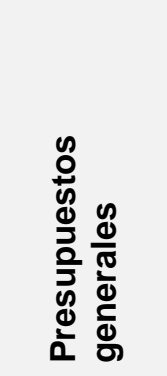 } & \multirow[b]{2}{*}{ 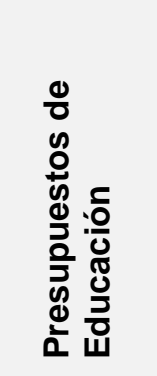 } & \multirow[b]{2}{*}{ ஃ } & \multicolumn{7}{|c|}{ Distribución de la partida para la educación pública } \\
\hline & & & & 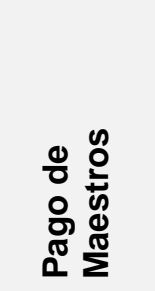 & 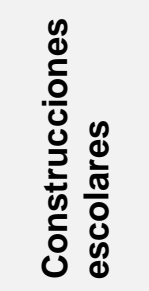 & 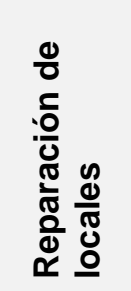 & 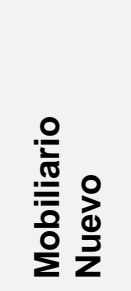 & 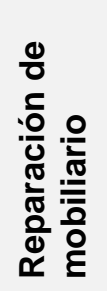 & 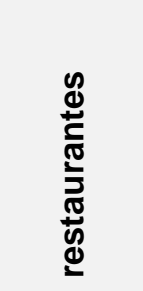 & $\begin{array}{l}\frac{n}{\pi} \\
\frac{\pi}{\frac{\pi}{\pi}} \\
\frac{\pi}{\sigma}\end{array}$ \\
\hline $4^{\mathrm{a}}$ & 106.68364 & 15.00669 & 14,06 & 4.72500 & 2.76700 & 90100 & 68100 & 7200 & 2.20600 & 3400 \\
\hline $5^{\mathrm{a}}$ & 110.66007 & 26.71298 & 24,13 & 4.15084 & 14.29784 & 1.24300 & 97500 & 7600 & 1.62500 & 95620 \\
\hline $6^{\mathrm{a}}$ & 95.31561 & 17. 42429 & 18,28 & 4.78533 & 5.11475 & 1.43900 & 57000 & 19200 & 1.56731 & 45300 \\
\hline Casanare & 24.15555 & 5.21285 & 21,5 & 1.68800 & 1.42791 & 110.00 & 15639 & 4000 & 54380 & 5800 \\
\hline \multirow[t]{2}{*}{ Total \$ } & 983.09614 & 139.04415 & 14,14 & 30.85717 & 54.83782 & 7.26853 & 3.98239 & 83900 & 15.41965 & 3.04120 \\
\hline & Porcentaje & & & 22,12 & 39,43 & 5,23 & 28,04 & 0,60 & 11,08 & 2,18 \\
\hline
\end{tabular}

Fuente: Información tomada del Informe del Director de Educación Pública al señor gobernador de Boyacá. Tunja, 1938.

Igualmente, la situación nacional y mundial no favoreció en mucho estos esfuerzos nacionales, que llevaron a los departamentos a aumentar los valores en las matriculas, reducir becas nacionales y departamentales para el ingreso a las escuelas normales, urbana-rural, y en muchos casos la suspensión de los salarios de maestros y maestras, como sucedió en Boyacá hacia el año de 1932, donde se presentó varias situaciones de protestas por parte del estudiantado y la población, como se muestra a continuación:

El gobernador de ese momento Silvino Rodríguez acudió a los recursos del
departamento para sostener la institución con sus propios recursos a través de las
matriculas a $\$ 20$ (antes a $\$ 4$ ); las pensiones de los alumnos internos a $\$ 20$ (antes
$\$ 18$ ); las matriculas de los alumnos de la escuela anexa a $\$ 5$ (antes $\$ 2$ o nada).
Además, se exigió a los alumnos externos una ayuda a los gastos generales de $\$ 2$
por mes y de los anexos $\$ 1$, (INFORME, 1931, p. 30 )

Esto para mantener en funcionamiento la estructura administrativa. Además, muchos docentes por voluntad colectiva continuaron clases con pequeños auxilios económicos que brindó el departamento.

Pero estas medidas económicas, no fueron acogidas por los estudiantes de grado $\mathrm{V}$, quienes organizaron protestas, contando con el apoyo de la población, por lo cual las directivas de la Institución Normal masculina se vieron obligados a aislarlos de otros cursos, para evitar protestas generalizadas. A su vez denunciaron los inconvenientes de desigualdad intelectual, y la necesidad de "de crear un curso preparatorio" (INFORME, 1931, p. 30), para que los estudiantes que ingresen a los diferentes niveles, estuvieran en las mismas condiciones académicas, logrando el aprovechamiento de su formación en áreas como matemática y física.

Por otra parte, los maestros y maestras promovieron el trabajo vocacional, para garantizar el funcionamiento de las Escuelas Normales en el departamento, evitando el cierre de las mismas. También buscaron el auxilio de las especializaciones, que no mitigaron ni ayudaron en el cumplimiento de estos pagos, con los cuales se sostendrían a estudiantes, maestros y maestras internas, personal administrativo, adquisición de 
materiales para biblioteca. Se presentaron situaciones donde estás instituciones dejaron de recibir por más de seis meses ingresos para poder responder a las necesidades de los y las estudiantes, como lo señalaron las directivas en uno de sus informes entregados al secretario de educación departamental: "El auxilio para los cursos de especialización en pedagogía y matemáticas no ha tenido efectividad en el pago y los alumnos tuvieron que soportar una situación bastante critica a pesar de la buena voluntad que siempre manifestó el señor director de Educación para que la caja del departamento cumpliera con su deber" (INFORME, 1932).

Asimismo, existió una preocupación por parte de los inspectores y secretarios de instrucción pública sobre las condiciones y la forma de aplicación de la reforma constitucional de 1936, específicamente el artículo 14, el cual autorizó al estado para que, "respetando el concepto de libertad de enseñanza, el gobierno pudiera intervenir en la marcha de la educación pública y privada, a fin de garantizar los fines sociales, de la cultura y la mejor preparación intelectual, moral y física de los educandos" (URIBE, 1989, p. 93), incitando a los choques y enfrentamientos entre la iglesia y los gobiernos liberales, por considerarse a este tipo de reformas como ideas de tendencias socialistas y comunista, que se venían propagando en toda la nación desde su llegada al poder en el año de 1930, criticando fuertemente a las poblaciones donde fueron acogidas a cabalidad como en los municipios de Chiquinquirá, Sogamoso, Moniquirá, que hacen parte de Boyacá.

Para contrarrestar el auge de estas ideas en la población e instituciones para la formación de educadores, se creó la ordenanza de propaganda sociológica, que inició en el departamento de Boyacá hacia el año de 1931. Tuvo como tarea la elevación de la moral, apoyando el resurgimiento de la liga de damas católicas colombianas ${ }^{21}$, que contempló entre otras cosas, la creación de cooperativas de ahorro y ligas que promovieron las buenas virtudes entre los pobladores, sobre todo en las mujeres quienes debían cultivar sus buenas virtudes. Se resaltó el trabajo desarrollado en Guateque por Luis Enrique Osorio, "quien fundó en la ciudad la legión Femenina del Alfabeto, institución que sustancialmente figuraba en la liga con el nombre de acción educativa y con el carácter de una de las secciones, ya que la liga abarca todas las actividades que la mujer puede desarrollar en el campo social" (URIBE, 1989, p. 52), convirtiéndose en una forma de control entre la población femenina ${ }^{22}$, muchas de ellas dedicadas al cuidado del hogar, monasterio y magisterio, como labores acordes a su condición biológica.

Para mejorar las condiciones de la población en edad escolar, que asistió a las escuelas rurales y campesinas se "propuso los programas de cobertura en materiales para las escuelas y servicios higiénicos para escolares. Además, se consideró de urgencia el desarrollo de las escuelas rurales y campesinas frente a las urbanas" (URIBE, 1989, p. 96), a través de la implementación de cartillas y métodos provenientes de la Escuela Nueva, que le trajo problemas con las autoridades eclesiásticas y locales, quienes interpretaron

${ }^{21}$ Institución que había antes funcionado como proyección de la que en Bogotá ha trabajado desde hace algún tiempo con brillantes resultados en todos los órdenes del campo social y que acaba de merecer del primado de Colombia un férvido elogió y una explicita recomendación para toda la república. Ver Informe (1931, p. 51).

22 Probablemente muchas de estas instituciones que funcionaron en el departamento, contando con el apoyo del párroco de cada municipio, se convertían en la única forma de combatir las ideas reformistas de una educación laica promovida por los gobiernos liberales, así como las ideas socialistas y renovadoras que llevaron al departamento de Boyacá a convertirse en uno de los epicentros de lucha y reivindicación de los derechos civiles, culturales y políticos de las mujeres en Colombia. Ver Valbuena Porras (2010, p. 55). 
estás medidas como Socialistas.

Así mismo, desde la dirección de Educación Pública, se intensificó la propaganda pedagógica con la difusión de la Revista Cultura, folletos y libros que permitieron llegar a toda la población boyacense, invitando a maestros y maestras, inspectores de educación, inspectores locales y médicos a que contribuyeran con la divulgación de estas ideas que contrarrestaron el analfabetismo y la ignorancia, pese a la resistencia que generó entre algunos grupos sociales la puesta en marcha de dichas reformas. Con ellas, se buscó la construcción de bibliotecas en todos los municipios del departamento ${ }^{23}$, se implementó la capacitación pedagógica de los docentes durante en horas laborales, se crearon los cursos vacacionales para maestros, aparecieron los liceos pedagógicos y conferencias de inspectores ${ }^{24}$.

\section{Las escuelas normales en Boyacá: retos y vicisitudes}

Las escuelas normales "eran el canal de movilidad social de las clases medias y las élites locales. Desde 1927 habían cambiado profundamente de orientación y la iglesia perdió su poder sobre ellas. En esta época no obstante, el cambio pareció menos radical porque las escuelas fueron confiadas a extranjeros" (HELG, 1980, p. 175), y para el caso de Boyacá, desde 1925 se habían contratado los servicios de Julius Sieber con el fin de reformar la Escuela Normal y poder llevar a esta institución "un grado superior de perfección y eficacia en la formación de los maestros para la enseñanza primaria en el departamento", convirtiéndose esta institución en "ciencia y virtud" para transformarse en "la entidad llamada a despertar en los alumnos maestros una sana y vigorosa inquietud mental" (INFORME, 1931, p. 13).

Para llevar a cabo los cambios necesarios en la Escuela Normal, Julius Sieber, en colaboración con Rafael Bernal25, generó la restructuración del pensum, la aplicación de un nuevo reglamento, la capacitación y cursos vacacionales para maestros rurales, anexos a

${ }^{23}$ Cada año se aumentó en estas bibliotecas nuevos volúmenes, escogidos por profesores competentes entra las obras de más autores relativas a la pedagogía y a la organización escolar. Ver Informe (1932, p. 12).

${ }^{24}$ Fueron creados como los medios más prácticos que establecen las disposiciones reglamentarias del ramo para procurar el cambio de ideas, el estudio y la discusión de temas de importancia para el ejercicio profesional del maestro. Los liceos pedagógicos municipales, formados por los directores de escuelas Urbanas y rurales, han constituido un medio de evidente utilidad en la resolución de los problemas de interés local que se presentan en la marcha de las escuelas y en la discusión de asuntos referentes a la práctica de la enseñanza. Entre las Nuevas instituciones destinadas a la preparación complementaria del maestro, de esta una de las que se han desarrollado con mayor interés, y aumenta considerablemente en su utilidad, toda vez que puede ser dirigida por el inspector seccional de educación quien dispone de la herramienta necesaria para resolver las cuestiones que son materia de consulta. Ver Informe (1932, p. 11).

25 Rafael Bernal como humanista, se destacó porque entre sus mayores preocupaciones estuvo "la dependencia de la educación Colombiana" a implementar reformas educativas que nada tenían que ver con las necesidades de la población. Esta situación que surgió desde "las minorías intelectuales con el deseo de perfección acudieron a ideologías políticas y sistemas europeos y norteamericanos para buscar el modelo aplicable a la nación ideal"; modelos y concepciones propias de su territorio, de sus realidades, por lo que allí no estaba la solución a la crisis de la escuela colombiana, la cual debía basarse tanto en los principios universales de la educación, como en "la realidad e un pueblo cuya histórica edad, cuya posición geográfica, cuya mezcla étnica, cuya heterogeneidad de climas, productos y accidentes topográficos lo presenta como características peculiares diversas a las que dominan otras latitudes", pero para poder generar aquella escuela democrática se debe dar importancia a la escuela rural, ya que no se puede olvidar que hasta la mitad del siglo 20 , la mayoría de la población estaba en las zonas rurales, con características propias de su contexto, a la cual no se podía obligar adaptarse a un medio Urbano con necesidades diferentes, porque era necesario crear y establecer una enseñanza agrícola y formar maestros para este campo. Ver Ocampo López (1999, p. 312). 
la Escuela Normal. Propuso que durante los primeros años escolares se impartieran todos los principios y teorías de la educación, para que en los dos años restantes se destinaran a la especialización en pedagogía, para ejercer como maestros de educación secundaria.

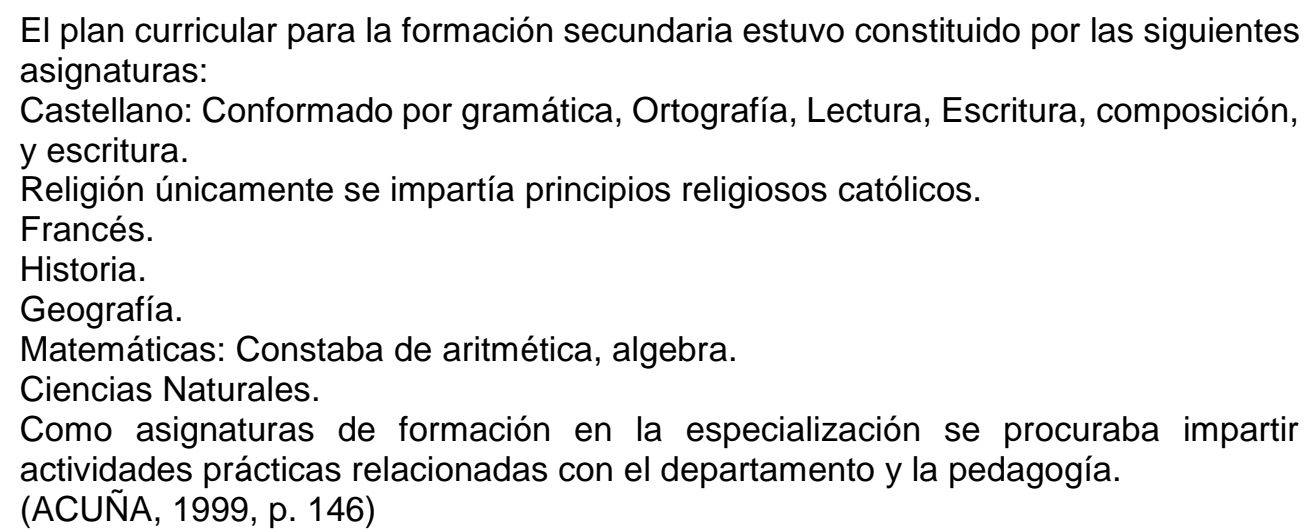

Con estas últimas asignaturas se buscó restar valores y tradiciones culturales con creatividad. El estimuló a los estudiantes para poder continuar sus estudios estaba en las becas nacionales y departamentales, que se asignaba a los estudiantes con excelente rendimiento, acorde a los resultados obtenidos por asignatura cada año. Entre los requisitos para ingresar a la escuela normal se encontraban los siguientes principios: ser católico, buena conducta, no poseer enfermedades contagiosas, no tener menos de 13 años, anexar certificados de educación primaria, presentar exámenes de admisión.

Con esto las reformas llevadas a cabo en el departamento de Boyacá no solo permitieron la formación y capacitación del estudiante, del maestro sino que reafirmó indirectamente el poder de la iglesia católica, cada vez que se exigió al alumnado profesar esta única religión. Probablemente esta postura fue el resultado de las mismas tendencias políticas que tenían los gobernantes locales y municipales.

Además de ello, Sieber adelanto cursos de especialización para maestros de segunda enseñanza, así como especializaciones en Pedagogía, matemáticas y ciencias Naturales.

Conscientes de las grandes necesidades existentes en el campo y por el tipo de población, se crearon a su vez Escuelas Normales Rurales "institutos especiales para la preparación abreviada de maestras rurales" (ESTATUTO, 1934), para llegar a las zonas aisladas del departamento, poder formar las nuevas generaciones a través de un pensum que comprendía dos años de estudio, el cual estuvo integrado por las siguientes materias:

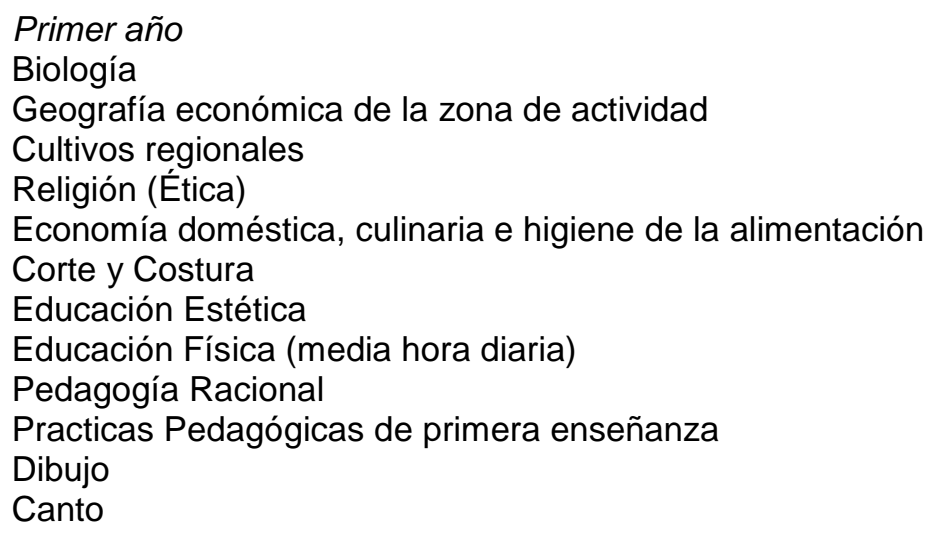


Segundo año

Higiene

Cultivos regionales

Elementos de contabilidad y comercio

Trabajos manuales

Dibujo

Educación Cívica

Educación Física

Arreglo, cuidado y estética del hogar

Metodología general de primera enseñanza

Prácticas pedagógicas de primera enseñanza.

(ESTATUTO DE LA NORMAL DE MAESTRAS RURALES, 1934, p. 10)

Para el caso de las maestras que se encontraban ejerciendo en zonas rurales, se crearon estímulos como becas de estudio durante vacaciones, con el fin de ampliar sus conocimientos en otras áreas como Física, formación pedagógica y nuevas prácticas metodológicas:

Este curso, al cual el año pasado concurrieron 107 alumnas maestras y éste se desarrolló en los años $1^{\circ}, 2^{\circ}$ y $3^{\circ}$ de acuerdo con el pensum respectivo y con las disposiciones del decreto orgánico, dictado por el gobernador con fecha 27 de octubre del año próximo pasado:

Artículo 1. El curso pedagógico de vacaciones para maestras de escuelas rurales correspondiente al presente año, se instalará el $1^{\circ}$ de diciembre próximo y se clausurará el último del mismo mes.

Artículo $2^{\circ}$. Habrá en este curso tres secciones que corresponderán a los tres años de estudios suplementarios establecidos por la reglamentación vigente.

Artículo $3^{\circ}$. El horario y el pensum de estudios corresponderán a lo prescrito por el artículo $3^{\circ}$ del decreto número 551 de 1927.

Artículo $4^{\circ}$. Las maestras que concurrieron el año pasado en calidad de alumnas becadas para las secciones 1 y 2, disfrutarán en I presente año del mismo beneficio, siempre que hubieren sido calificadas satisfactoriamente, que hayan servido a la escuela en el mismo año y que su conducta y consagración hayan sido satisfactorias, a juicio del respectivo inspector. Podrán igualmente concurrir hasta vente alumnas supernumerarias.

Artículo $5^{\circ}$. Para ingresar al curso pedagógico de vacaciones, se requiere ser maestras de escuela rural en ejercicio, tener menos de treinta y cinco años, haberse inscrito ante el respectivo inspector provisional de educación y haber sido llamada por la dirección del ramo. Informe, Informe del director de educación Pública al gobernador del departamento. (TUNJA: IMPRENTA DEL DEPARTAMENTO, 1932, p. 9)

Las Escuelas Normales Anexas, también recibieron apoyo institucional, fueron reestructurados sus planes de estudio, contando con los esfuerzos locales y departamentales. De igual forma, funcionaron las Escuelas Normales Agrícolas, las cuales contaron desde sus inicios, con la ayuda de los padres de familia, quienes muchas veces hicieron donaciones de terreno para que los estudiantes lograrán hacer las prácticas pertinentes, que pondrían en conocimiento, luego de graduados con niños y niñas en escuelas de primaria y secundaria de las zonas urbanas y rurales del departamento.

Si bien, todos los esfuerzos por consolidar la creación y transformación de las escuelas en las zonas urbanas y rurales para la formación primaria, secundaria y normalista, contaron con los esfuerzos locales, municipales, y departamentales, fueron constantes las quejas por la carencia de equipos, inmuebles e infraestructura adecuada que facilitará el ejercicio docente en estas instituciones, así como la falta de granjas escolares, fundamentales para la enseñanza y prácticas agrícolas. Pese a ello, durante los 
años de 1930 a 1938 existió un aumento en los índices de alfabetización y el número de estudiantes matriculados en primaria, secundaria e instituciones normalistas se incrementó notablemente.

\section{Escolarización en el Departamento de Boyacá}

Los cambios generados por mejorar las escuelas normales en el Departamento, contribuyeron indirectamente en la creación y reestructuración de las instituciones de primaria y secundaria, las cuales recibieron más estudiantes, tuvieron mejores maestros y maestras capacitadas, se legisló a favor de una educación obligatoria para todos y todas, lo que incrementó la asistencia de los niños y niñas a las escuelas rurales y Urbanas, se implementaron programas de nutrición, agricultura, medicina escolar e higiene, como se muestra a continuación.

Tabla 3 - Número de escuelas y número de estudiantes matriculados.

\begin{tabular}{|c|c|c|c|c|c|c|c|c|c|c|c|c|c|c|}
\hline \multicolumn{15}{|c|}{ Número de escuelas totales por el Departamento de Boyacá - 1932} \\
\hline \multicolumn{5}{|c|}{ Urbanas } & \multicolumn{5}{|c|}{ Rurales } & \multicolumn{5}{|c|}{ Totales } \\
\hline $\begin{array}{c}\text { De } \\
\text { varones }\end{array}$ & $\begin{array}{c}\text { De } \\
\text { niñas }\end{array}$ & \multicolumn{2}{|c|}{$\begin{array}{l}\text { Alter- } \\
\text { nadas }\end{array}$} & Total & $\begin{array}{c}\mathrm{De} \\
\text { varones }\end{array}$ & $\begin{array}{c}\text { De } \\
\text { niñas }\end{array}$ & \multicolumn{2}{|c|}{$\begin{array}{l}\text { Alter- } \\
\text { nadas }\end{array}$} & Total & $\begin{array}{c}\mathrm{De} \\
\text { varones }\end{array}$ & $\begin{array}{c}\text { De } \\
\text { niñas }\end{array}$ & \multicolumn{2}{|c|}{$\begin{array}{l}\text { Alter- } \\
\text { nadas }\end{array}$} & Total \\
\hline 165 & 135 & \multicolumn{2}{|c|}{2} & 302 & 18 & 13 & \multicolumn{2}{|c|}{374} & 405 & 183 & 148 & \multicolumn{2}{|c|}{376} & 707 \\
\hline \multicolumn{15}{|c|}{ Número de alumnos matriculados } \\
\hline \multicolumn{5}{|c|}{ Urbanas } & \multicolumn{5}{|c|}{ Rurales } & \multicolumn{5}{|c|}{ En todas las escuelas } \\
\hline $\begin{array}{c}\text { De } \\
\text { varones }\end{array}$ & \multicolumn{2}{|c|}{ De niñas } & \multicolumn{2}{|c|}{ Totales } & $\begin{array}{c}\mathrm{De} \\
\text { varones }\end{array}$ & De ni & & \multicolumn{2}{|c|}{ Totales } & $\begin{array}{c}\mathrm{De} \\
\text { varones }\end{array}$ & \multicolumn{2}{|c|}{ De niñas } & \multicolumn{2}{|c|}{ Totales } \\
\hline 13451 & \multicolumn{2}{|c|}{8640} & \multicolumn{2}{|c|}{22091} & 14192 & \multicolumn{2}{|c|}{8993} & \multicolumn{2}{|c|}{23185} & 27585 & \multicolumn{2}{|c|}{17601} & \multicolumn{2}{|c|}{45186} \\
\hline \multicolumn{15}{|c|}{ Número de escuelas totales por el Departamento de Boyacá - 1937} \\
\hline \multicolumn{5}{|c|}{ Urbanas } & \multicolumn{5}{|c|}{ Rurales } & \multicolumn{5}{|c|}{ Totales } \\
\hline $\begin{array}{c}\text { De } \\
\text { varones }\end{array}$ & $\begin{array}{c}\text { De } \\
\text { niñas }\end{array}$ & \multicolumn{2}{|c|}{$\begin{array}{l}\text { Alter- } \\
\text { nadas }\end{array}$} & Total & $\begin{array}{c}\text { De } \\
\text { varones }\end{array}$ & $\begin{array}{c}\text { De } \\
\text { niñas }\end{array}$ & \multicolumn{2}{|c|}{$\begin{array}{l}\text { Alter- } \\
\text { nadas }\end{array}$} & Total & $\begin{array}{c}\text { De } \\
\text { varones }\end{array}$ & $\begin{array}{c}\text { De } \\
\text { niñas }\end{array}$ & \multicolumn{2}{|c|}{$\begin{array}{l}\text { Alter- } \\
\text { nadas }\end{array}$} & Total \\
\hline 159 & 136 & \multicolumn{2}{|c|}{3} & 298 & 10 & 5 & \multicolumn{2}{|c|}{322} & 337 & 169 & 141 & & & 635 \\
\hline & & & & & úmero de & alumn & & & ulados & & & & & \\
\hline & Urba & las & & & & Rura & & & & & Tota & & & \\
\hline $\begin{array}{c}\text { De } \\
\text { varones }\end{array}$ & De ni & ias & & otal & $\begin{array}{c}\text { De } \\
\text { varones }\end{array}$ & De ni & & & otal & $\begin{array}{c}\text { De } \\
\text { varones }\end{array}$ & De ni & & & Total \\
\hline 9929 & 773 & & & 7659 & 10709 & 728 & & & 7995 & 11008 & 150 & & & 6024 \\
\hline
\end{tabular}

Fuente: Informe (1932 y 1937).

En la tabla $n .1$ el aumento de escuelas y estudiantes está directamente relacionado a las políticas educativas ejecutadas por los gobiernos liberales, quienes además de procurar por garantizar la formación e idoneidad del maestro, buscaron generar escuelas, aumentando en un "17. 6\% las escuelas rurales, así como las matriculas a nivel nacional en un 18\%" (HELG, 1980, p. 19), incrementó que prevaleció hasta finales de 1936, cuando se intensificaron los conflictos entre la iglesia y el estado por la libertad de enseñanza, así como la agudización de los conflictos bipartidistas por la búsqueda de una educación 
adecuada para la población colombiana. Sin embargo, esto no fue obstáculo para que los municipios del Departamento de Boyacá

\begin{abstract}
respondieran con fervor a los esfuerzos y a la política trazada por la nación y el departamento en punto de atención a la cultura...se destina para la educación pública $\$ 547.177-12$, lo que representa un poco más de la cuarta parte del presupuesto total, cosa que muy difícilmente puede ostentar otro departamento de la República. Pero no es esto solo sino que ha habido un criterio directriz en la inversión de dichos fondos. En efecto de los \$547.000-00 se han destinado \$320000-00 para el pago de sueldos y pensiones del magisterio; $\$ 32.000-00$ para restaurantes escolares; $\$ 30.000-00$ para construcciones escolares; $\$ 21.000-00$ para internados agrícolas; $\$ 33.000-00$ para la campaña de sanidad escolar; $\$ 11.000-00$ para la inspección de enseñanza primaria, y los $\$ 100.000-00$ restantes se invierten en servicios tan importantes como el sostenimiento del colegio departamental femenino, de la escuela Normal Rural, de la Escuela Profesional para obreros, del Instituto Alfonso López, de la escuela bolivariana, de los centros culturales obreros, de la campaña de cinematografía educativa y del personal administrativo de la secretaria de educación. (RUBIANO, 1938, p. 41)
\end{abstract}

Estas circunstancias, reafirmaron la existencia clara de políticas educativas departamentales para fomentar la creación y formación de maestros, con estímulos a través de becas nacionales, departamentales y municipales para garantizar la educación de niños y niñas de establecimientos urbanos y rurales. Se crearon iniciativas culturales, el fomento de internados agrícolas para fomentar el valor a la tierra y recursos naturales que posee el campo boyacense, estímulo a la enseñanza complementaria y normalista.

\title{
Conclusión
}

Para terminar, estos gobiernos adelantaron legislación a favor de la situación de los trabajadores y de las mujeres, lograron estructurar y fortalecer políticas educativas para eliminar el analfabetismo con los nuevos modelos pedagógicos. Aunque su impacto estuvo dado a largo plazo, por las dificultades que tuvo la misma sociedad colombiana para legitimar y transformar los sistemas administrativos, sociales, culturales heredados de la colonia, consolidaron mecanismos hacia una nación igualitaria, en la búsqueda de una modernidad pensada para toda una nación, con el fortalecimiento del escenario sociopolítico de los movimientos sociales.

En el caso del departamento de Boyacá, las reformas implementadas por los gobiernos liberales no tuvieron la acogida esperada, contando con la vigilancia y control de la Iglesia Católica y el Partido Conservador, quienes siempre la consideraron como una amenaza para la organización del sistema educativo que solo trajo la perdida de los buenos valores morales, cristianos y el fortalecimiento del ateísmo, acorde a las libertades promulgadas para toda la población. Sin embargo, logró consolidar un presupuesto educativo con el cual buscó mantener los centros médicos y de higiene en las instituciones, restaurantes escolares, cine y teatro, bibliotecas, así como el estímulo de una educación complementaria con la creación de escuelas normales tanto urbanas como rurales para la formación de maestros y maestras acorde a las necesidades del departamento y la nación, con el fin de contrarrestar los índices de analfabetismo que tuvo el departamento durante el periodo de estudio.

Ejemplo de ello, fueron los esfuerzos por la construcción de la Escuela Normal Nacional, que tuvo capacidad para 900 estudiantes, garantizando la formación de 
generaciones de boyacenses y estudiantes provenientes de otros departamentos como Santander, Tolima, Norte de Santander, Meta, entre otros. Contaron con el acceso a instituciones de educación superior que complementaron su educación en áreas del conocimiento como: Cultura Física, Bellas Artes, Economía, Veterinaria, Medicina, Enfermería, carreras a las cuales accedieron por medio de becas nacionales y departamentales.

Se generaron políticas educativas en la reorganización de las escuelas normales rurales, creadas por el departamento desde 1925 y consolidadas en el periodo de hegemonía liberal, llegando a aquellos maestros que ejercieron en zonas rurales sin contar con títulos que los acreditaran en su ejercicio profesional. Tanto las zonas rurales como urbanas contaron con programas de higiene y sanidad escolar, restaurantes, granjas escolares, excursiones, etc.

\section{Referencias}

ACUÑA, Blanca Ofelia. La Escuela Normal de Institutores y la segunda misión alemana. In: COLOQUIO DE HISTORIA DE LA EDUCACIÓN COLOMBIANA, 3, 1999. Actas... Popayán: Universidad del Cauca, 1999, p. 146-147.

ÁLVAREZ GALLEGO, Alejandro. Y la escuela se hizo necesaria: en busca del sentido actual de la escuela. Bogotá: Magisterio-Sociedad Colombiana de Pedagogía,1995.

ASAMBLEA DE BOYACÁ. Proyecto de Ordenanza, 1925.

BAUTISTA, Sandra Patricia Santamaría. La familia tunjana 1930-1946: hegemonia liberal. 2009. 246f. Tesis (Maestría en Historia) - Universidad Pedagógica y Tecnológica de Colombia, Tunja, 2009.

BORDA FALS. Regionalización y educación primaria en áreas rurales de Boyacá. UPTC: La Rana y El Águila, 1976.

BOYACÁ. Asamblea departamental. Boyacense 25 mayo 1934. Legajo 068.

. Decreto n. 531 de 1934. Boyacense 16 nov. 1934. Legajo 074.

. Dirección de Instrucción Pública. Decreto n. 309 de 1933. En el periódico Boyacense 28 nov. 1933. Legajo 071.

. Dirección de Instrucción Pública. Decreto n. 401 de 1936. En el periódico Boyacense del 1 oct. 1936. Legajo 066.

. Dirección de Instrucción Pública. Decreto n. 508 de 1936. En el Boyacense del 31 de diciembre de 1936. Legajo 067.

CIDER-CENTRO INTERDICIPLINARIO DE INVESTIGACIONES SOBRE DESARROLLO. La nueva ruralidad. Serie documentos conceptuales. Bogotá: Universidad de los Andes, 2000.

CORTS GINER, María Isabel; ÁVILA FERNÁNDEZ, Alejandro; CALDERÓN, María Consolación; MONTERO, Ana María. Historia de la educación: cuestiones previas y perspectivas actuales. España: Gipes, 1996.

DECRETO N. 110 DE 1939. Boyacá: Secretaria de Educación.

DECRETO N. 325 DE 1939. Boyacá: Secretaria de Educación.

DECRETO N. 401 DE 1936. Boyacá: Secretaria de Educación.

DECRETO N. 89 DE 1932. Boyacá: Secretaria de Educación. 
ESTATUTO DE LA NORMAL DE MAESTRAS RURALES. Bogotá: Imprenta Nacional, 1934.

GINER, María Isabel Corts; FERNÁNDEZ, Alejandro Ávila María; CALDERÓN, Consolación; MONTERO, Ana María. Historia de la educación: cuestiones previas y perspectivas actuales. España: Gipes, 1996.

HELG, Aline. La educación en Colombia 1910-1957: una historia social, económica y política. Bogotá: Ccerec, 1987.

. La educación primaria y secundaria durante el primer gobierno de Alfonso López Pumarejo (1934-1938). Revista Colombiana de Educación, Bogotá, n. 6, p. 1-22, 1980.

HERRERA, Martha Cecilia. Modernización y escuela nueva en Colombia. Bogotá: Plaza y Janes, 1999.

Historia de la educación en Colombia: la república liberal y la modernización de la educación 1930-1946. Revista Colombiana de Educación, Bogotá, n. 26, 1993.

INFORME DEL DIRECTOR DE EDUCACIÓN PÚBLICA AL GOBERNADOR DEL DEPARTAMENTO. Tunja: Imprenta del departamento, 1931.

INFORME DEL DIRECTOR DE EDUCACIÓN PÚBLICA AL GOBERNADOR DEL DEPARTAMENTO. Tunja: Imprenta del departamento, 1932.

INFORME DEL DIRECTOR DE EDUCACIÓN PÚBLICA AL GOBERNADOR DEL DEPARTAMENTO. Tunja: Imprenta del departamento, 1933.

INFORME DEL DIRECTOR DE EDUCACIÓN PÚBLICA AL GOBERNADOR DEL DEPARTAMENTO. Tunja: Imprenta del departamento, 1935.

INFORME DEL DIRECTOR DE EDUCACIÓN PÚBLICA AL GOBERNADOR DEL DEPARTAMENTO. Tunja: Imprenta del departamento, 1938.

INFORME DEL DIRECTOR DE LA ESCUELA NORMAL DE INSTITUTORES. Tunja, 1932. Fondo posada, libro 12923.

LONDOÑO, Carlos Arturo. Educación en la democracia integral: el constructivismo práctico de John Dewey y el impacto pedagógico en la escuela nueva durante la república liberal en Colombia. Cuestiones de Filosofía, n. 6, p. 22-23, 2004.

MEMORIAS DE LOS SECRETARIOS DE EDUCACIÓN EN BOYACÁ. Tunja: Imprenta Departamental, 1931.

NIETO CABALLERO, Agustín. Bases para la educación. Legajo 112.

OCAMPO LÓPEZ, Javier. Rafael Bernal Jiménez: sus ideas educativas y la escuela nueva en Boyacá. COLOQUIO DE HISTORIA DE LA EDUCACIÓN COLOMBIANA, 3, 1999. Actas Popayán: Universidad del Cauca, 1999.

Rafael Bernal Jiménez: sus ideas educativas, sociológicas, humanistas y la escuela nueva en Boyacá. Tunja: Rudecolombia, 2001.

PARRA SANDOVAL, Rodrigo. Escuela y modernidad en Colombia: la escuela rural. Bogotá: Fundación FES, 1996.

PARRA, Lina Adriana. Los orígenes de la Universidad Pedagógica y Tecnológica de Colombia. Revista Historia de la Educación Latinoamericana, n. 6, p. 167-168, 2008.

RAMÍREZ, Alba Nidia Triana. Historia de la educación primaria rural en Colombia 19461994. 2008. Tesis (Doctorado en Educación) - Universidad Pedagógica y Tecnológica de Colombia, Tunja, 2008.

RAMÍREZ, María Teresa; TELLEZ, Juana Patricia. La educación primaria y secundaria en 
Colombia en el siglo 20. Bogotá: Editorial Banco de la República, 2006. Tomado de: http://www.banrep.gov.co/sites/default/files/publicaciones/pdfs/borra379.pdf

RUBIANO, Gonzalo Vargas. Informe del director de educación pública al señor gobernador de Boyacá. Tunja: Imprenta Departamental, 1938.

RUDECOLOMBIA CADE CARTAGENA. Educación y cultura retos del nuevo siglo en latinoamérica. Cartagena: Unicartagena, 2013.

SANTAMARÍA BAUTISTA, Sandra Patricia. La familia tunjana 1930-1946: hegemonia liberal. 2009. Tesis (Doctorado en Educación) - Universidad Pedagógica y Tecnológica de Colombia, Bogotá, 2009.

SIEBER, Julius. Proyecto sobre la escuela normal. Legajo 201.

SOTO ARANGO, Diana Elvira. La ruralidad en la cotidianidad escolar colombiana: historia de vida de la maestra rural boyacense 1948-1974. Revista Historia de la Educación Colombiana, Tunja, UPTC, n. 18, 2012, p. 213-216.

TRIANA RAMÍREZ, Alba Nidia. Historia de la educación primaria rural en Colombia 19461994. Tunja: UPTC, 2008. 390f. Tesis (doctorado en Educación). Universidad Pedagógica y Tecnológica de Colombia.

URIBE, Jaime Jaramillo. La educación durante los gobiernos liberales 1930-1946. In: MEJÍA, Álvaro Tirado. Nueva historia de Colombia. Bogotá: Planeta, 1989, p. 83-97.

VALBUENA PORRAS, Mónica Liset. Historia de vida: la maestra Flor de Orozco. Revista Historia de la Educación Colombiana, Universidad de Nariño, n. 17, p. 275-278, 2014.

. Historia de vida de la maestra Gloria Inés Prieto Duarte 1947-2003. 2013. Tesis

(Doctorado en Ciencias de la Educación) - Universidad de Cartagena, Cartagena, 2013.

. Lucha y organización de las mujeres en Boyacá 1930-1957. 2010. 92f. Tesis

(Monográfica para optar el título de licenciada en Ciencias Sociales) - Universidad Pedagógica y Tecnológica de Colombia, Tunja, 2010.

ZULUAGA GARCÉS, Olga Lucía. Hacia la construcción de un campo conceptual plural y abierto para la pedagogía: aproximaciones metodológicas. In: La investigación como práctica pedagógica, memoria del simposio internacional de investigadores en educación. Santafé de Bogotá: Convenio Andrés Bello, 2000, p. 35-57.

MÓNICA LISET VALBUENA PORRAS es Licenciada en Ciencias Sociales y candidata a magister en Historia de la Universidad Pedagógica y Tecnológica de Colombia.

Endereço: 150001 - Calle 3, n. 12-04 - Tunja - Boyacá - Colombia.

E-mail: monca3108@gmail.com

Recebido em 12 de agosto de 2015.

Aceito em 13 de março de 2016. 